\title{
Image Scanning Microscopy
}

\author{
Claus B. Müller and Jörg Enderlein* \\ III. Institute of Physics, Georg August University, 37077 Göttingen, Germany
}

(Received 28 October 2009; published 10 May 2010)

\begin{abstract}
A new microscopy technique is introduced, image scanning microscopy (ISM), which combines conventional confocal-laser scanning microscopy with fast wide-field CCD detection. The technique allows for doubling the lateral optical resolution in fluorescence imaging. The physical principle behind ISM is similar to structured illumination microscopy, by combining the resolving power of confocal-laser scanning microscopy with that of a wide-field imaging microscopy. This Letter describes the theoretical foundation and experimental realization of ISM.
\end{abstract}

DOI: 10.1103/PhysRevLett.104.198101

PACS numbers: $87.64 . \mathrm{kv}, 87.57 . \mathrm{cf}$

Classical fluorescence microscopy is limited in resolution by the wavelength of light (diffraction limit) restricting lateral resolution to ca. $200 \mathrm{~nm}$, and axial resolution to ca. $500 \mathrm{~nm}$ (at typical excitation and emission wavelengths around $500 \mathrm{~nm}$ ). The origin of this limit of spatial resolution is the finite support of the optical transfer function (OTF) of a microscope's optics, limiting the maximum Fourier mode that is still transmitted by the optics (for an excellent review, see Ref. [1]). However, recent years have seen a tremendous development in high- and superresolution techniques of fluorescence microscopy, pushing spatial resolution to its diffraction-dictated limits and much beyond, see reviews [1-6].

One of these techniques is structured illumination microscopy (SIM). In SIM, the sample is illuminated with a spatially modulated excitation intensity distribution (EID), and the emerging fluorescence is imaged with a conventional wide-field imaging setup. By moving and rotating the EID pattern in different positions and orientations, taking each time a wide-field image, a final fluorescence image is composed which has roughly double the resolution (laterally) of a conventional wide-field or a confocallaser scanning image alone [7]. With SIM, a roughly twofold enhancement in lateral spatial resolution is achievable. SIM has shown to achieve video-rate live cell imaging [8].

To better understand the origin of this resolution enhancement, let us consider image formation in SIM. For the sake of simplicity, we assume an imaging system that images, with unity magnification, fluorescent emitters within the focal plane of the optics onto a CCD camera placed at the conjugated focal plane. Choosing unity magnification simplifies all following mathematics because we do not have to distinguish between position in the sample and position on the CCD. Also, we restrict ourselves to considering imaging of a planar sample in the focal plane only. However, all the subsequent considerations are straightforward to extend to the general three-dimensional case with arbitrary magnification. Here, the recorded SIM image $I\left(\mathbf{r}, \mathbf{k}_{e}\right)$ is a function of position $\mathbf{r}$ on the imaging CCD and of the wave vector $\mathbf{k}_{e}$ describing the EID spatial modulation which is assumed to be of the form $1+$ $\cos \left(\mathbf{k}_{e} \cdot \mathbf{r}\right)$. Under ideal conditions, this image is then given by the convolution

$$
I\left(\mathbf{r}, \mathbf{k}_{e}\right)=\int d \mathbf{r}^{\prime} U\left(\mathbf{r}-\mathbf{r}^{\prime}\right)\left[1+\cos \left(\mathbf{k}_{e} \cdot \mathbf{r}^{\prime}\right)\right] c\left(\mathbf{r}^{\prime}\right)
$$

where $U\left(\mathbf{r}-\mathbf{r}^{\prime}\right)$ is the point-spread function (PSF) of wide-field imaging which describes the chance that a photon emitted from a molecule at position $\mathbf{r}^{\prime}$ in sample space is detected at position $\mathbf{r}$ on the $\mathrm{CCD}$, and $c(\mathbf{r})$ is the spatial density of fluorescent emitters in the focal plane. Switching to Fourier space yields

$$
\tilde{I}\left(\mathbf{k}, \mathbf{k}_{e}\right)=\tilde{U}(\mathbf{k})\left\{\tilde{c}(\mathbf{k})+\frac{1}{2}\left[\tilde{c}\left(\mathbf{k}+\mathbf{k}_{e}\right)+\tilde{c}\left(\mathbf{k}-\mathbf{k}_{e}\right)\right]\right\} .
$$

In a conventional wide-field microscope (no spatially structured illumination, i.e., $\mathbf{k}_{e}=0$ in the above equations), the finite resolution of imaging results from the finite support of the Fourier-transformed PSF (OTF) in $\mathbf{k}$ space, which means that there is a maximum length of the $\mathbf{k}$ vector beyond which the OTF vanishes. This maximum length is determined by the numerical aperture (NA) of the imaging system via

$$
\max (|\mathbf{k}|)=k_{\max }^{\mathrm{em}}=2 \pi \mathrm{NA} / \lambda_{\mathrm{em}},
$$

where $\lambda_{\mathrm{em}}$ is the vacuum wavelength of fluorescence. Thus, a conventional wide-field microscope cannot transmit any information on spatial Fourier components of the fluorophore distribution $c(\mathbf{r})$ having wave vector length larger than $k_{\max }^{\mathrm{em}}$, restricting the maximum resolvable spatial detail to a minimum size of $2 \pi / k_{\max }^{\mathrm{em}}=\lambda_{\mathrm{em}} / \mathrm{NA}$, which is the famous Abbe limit of resolution in wide-field microscopy. However, as can be seen from inspecting Eq. (2), SIM allows us to extend the maximum length of extractable Fourier components of $\tilde{c}(\mathbf{k})$ to $k_{\max }^{\mathrm{em}}+k_{e}$. The maximum achievable wave vector $k_{e}$ of spatial modulation of the EID is given by a similar equation as Eq. (3) but now at the excitation wavelength $\lambda_{\mathrm{ex}}$. Thus, the minimum size of spatial detail that can be extracted with SIM is given by 
the harmonic mean $\left(\lambda_{\mathrm{em}}^{-1}+\lambda_{\mathrm{ex}}^{-1}\right)^{-1} / \mathrm{NA}$ (assuming the same value of NA both for the excitation as well as the emission wavelength) which is roughly 2 times smaller than that of a conventional wide-field microscope.

For extracting all possible Fourier modes of $\tilde{c}(\mathbf{k})$ in SIM, it is necessary to record many images with different orientation of $\mathbf{k}_{e}$ for covering, as much as possible, the whole Fourier space in an isotropic manner. In the present Letter, we propose and demonstrate an alternative approach, which is based on a conventional confocal-laser scanning microscope. It uses the fact that a diffraction-limited laser focus is a special type of structured illumination carrying all the possible Fourier modes allowed by the NA of an objective. In contrast to a conventional confocal-laser scanning microscope (CLSM), which employs a point detector for fluorescence detection, our approach images the fluorescence light which is excited within the laser focus onto a position-sensitive CCD. Thus, for each position of the scanning focus, a whole image is recorded, so that the method will be called image scanning microscopy or ISM. The image seen by the CCD depends now on both the position $\mathbf{s}$ on the CCD as well as the scan position $\mathbf{r}$ in the sample. Using a similar notation as before, this image is given by

$$
I(\mathbf{r}, \mathbf{s})=\int d \mathbf{r}^{\prime} U\left(\mathbf{r}-\mathbf{r}^{\prime}+\mathbf{s}\right) E\left(\mathbf{r}-\mathbf{r}^{\prime}\right) c\left(\mathbf{r}^{\prime}\right)
$$

where the function $E(\mathbf{r})$ describes the EID within a laser focus with position $\mathbf{r}$. Thus, the full data set after one twodimensional scan of the sample consists now of the fourdimensional array of data $I(\mathbf{r}, \mathbf{s})$ being a function of two two-dimensional vectors $\mathbf{r}$ and $\mathbf{s}$. From these data, an effective image can be constructed by integrating $I(\mathbf{r}-$ $\mathbf{s} / 2, \mathbf{s})$ over $\mathbf{s}$, thus yielding

$$
I_{\mathrm{eff}}(\mathbf{r})=\int d \mathbf{s} I\left(\mathbf{r}-\frac{\mathbf{s}}{2}, \mathbf{s}\right)=\int d \mathbf{r}^{\prime} U_{\mathrm{eff}}\left(\mathbf{r}-\mathbf{r}^{\prime}\right) c\left(\mathbf{r}^{\prime}\right)
$$

Here, we have introduced an apparent or effective PSF, $U_{\text {eff }}(\mathbf{r})$, which can be found, by using Eqs. (4) and (5), to be equal to

$$
U_{\text {eff }}(\mathbf{u})=4 \int d \mathbf{v} U(2 \mathbf{v}) E(2 \mathbf{u}-2 \mathbf{v}) .
$$

Equation (6) shows that $U_{\text {eff }}(\mathbf{u})$ is equal to the original PSF convolved with the EID and then rescaled to half size. In Fourier space, this reads

$$
\tilde{U}_{\mathrm{eff}}(\mathbf{k})=2 \tilde{U}(\mathbf{k} / 2) \tilde{E}(\mathbf{k} / 2)
$$

Thus, similar to SIM, the effective image $I_{\text {eff }}(\mathbf{r})$ contains information of spatial details twice as small as those seen by a conventional wide-field microscope with an OTF $\tilde{U}(\mathbf{k})$

Ideally, for directly doubling the resolution of the CLSM, one would like to have an effective PSF which is simply the original PSF but rescaled by a factor of 2 ,
$U_{\text {eff }}(\mathbf{r})=U(2 \mathbf{r})$, or equivalently for the OTF $\tilde{U}_{\text {eff }}(\mathbf{k})=$ $2 \tilde{U}(\mathbf{k} / 2)$. One may achieve that in the following way: (i) transforming the image $I_{\text {eff }}(\mathbf{r})$ into Fourier space, thus obtaining $\tilde{I}_{\text {eff }}(\mathbf{k})$, (ii) applying an appropriate filter function $\tilde{W}(\mathbf{k})$, i.e., calculating $\tilde{W}(\mathbf{k}) \tilde{I}_{\text {eff }}(\mathbf{k})$, and (iii) backtransforming the result to real space. If we assume, for simplicity, that both the PSF as well as the EID have the same support in Fourier space, a suitable Fourier filter function would be

$$
\tilde{W}(\mathbf{k})=E^{-1}\left(\frac{\mathbf{k}}{2}\right) .
$$

However, such a choice of filter function has the problem that it diverges towards infinity the closer $\mathbf{k}$ comes to the edge of the support of $E\left(\frac{\mathbf{k}}{2}\right)$ in $\mathbf{k}$ space. This can lead to an unwanted amplification of image noise at spatial frequencies close to those divergences (ringing effects). To avoid such ringing effects, a better choice for $\tilde{W}(\mathbf{k})$ is

$$
\tilde{W}(\mathbf{k})=\frac{U\left(\frac{\mathbf{k}}{2}\right)}{\left[\epsilon+U\left(\frac{\mathbf{k}}{2}\right) E\left(\frac{\mathbf{k}}{2}\right)\right]}
$$

where $\epsilon$ is chosen sufficiently small, and $\tilde{W}(\mathbf{k})$ is set to zero for all values of $\mathbf{k}$ for which $\tilde{U}(\mathbf{k}) \tilde{E}(\mathbf{k})$ is smaller than $\epsilon$. In the experimental realization of ISM as discussed below, $\epsilon$ was set to 0.01 of the maximum value of $\tilde{U}(\mathbf{k}) \tilde{E}(\mathbf{k})$.

The experimental setup of the ISM system is shown in Fig. 1. Fluorescence excitation (1) is done by a supercontinuum white light laser source $(\lambda=470-700 \mathrm{~nm})$, equipped with an eight-channel acousto-optic tunable filter (Crystal Technology, Inc., Palo Alto, CA) followed by an optical filter for sideband reduction (FF01-390/482/563/ 640-25, Semrock, Rochester, NY). The excitation light is guided, through a single mode optical fiber, towards the microscope. After exiting the fiber and recollimation, the light is reflected towards the microscope's objective (UPLAPO 60x W, 1.2 N.A., Olympus Europe, Hamburg, Germany) (6) by a quad-band dichroic mirror (zt405/488/ 561/640rpc, Chroma Technology Corp, Rockingham, VT) (3). For sample scanning, a piezo scan mirror is used (S334.1SL, Physik In-strumente (PI) GmbH \& Co. KG,

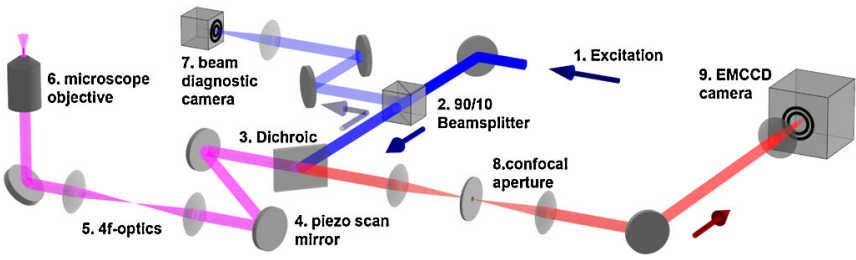

FIG. 1 (color online). ISM Setup, (1) Excitation with supercontinuum white light source and acousto-optic tunable filter, (2) 90/10 nonpolarizing beam splitter cube, (3) major dichroic mirror, (4) piezo scan mirror, (5) $4 f$ telescope, (6) UPL APO 60x $\mathrm{W}$ microscope objective, (7) beam diagnostic camera, (8) confocal aperture, and (9) EM CCD detection camera system. 
Karlsruhe, Germany) (4) in front of a $4 f$-telescope lens system (5). Fluorescence is collected by the same objective and guided back through the $4 f$ optics, descanned by the piezo scan mirror, and transmitted through the dichroic mirror. After passing an emission filter (FF01-446/523/ 600/677-25, Semrock, Rochester, NY), the fluorescence light is focused through a circular confocal aperture (diameter $200 \mu \mathrm{m})(8)$, recollimated, and imaged onto an electron multiplying (EM) CCD camera (iXon DU860-D, Andor Technology plc, Belfast, UK) (9). For the chosen magnification, the pixel size of the EM CCD corresponds to $80 \mathrm{~nm}$ by $80 \mathrm{~nm}$ in sample space. This is slightly smaller than the maximum achievable ISM resolution and large enough to provide reasonably good signal-to-noise ratio [9]. Ten percent of the backscattered light from sample area is directed, by a 90/10 beam splitter (Walter Uhl, Technische Mikroskopie GmbH \& Co. KG, Aßlar, Germany) (2), towards a CMOS camera (Guppy F-036B, Allied Vision Technologies GmbH, Stadtroda, Germany) for alignment and beam diagnostics.

When scanning a sample with the ISM system, the result is a discrete data set $I\left(\mathbf{r}_{j k}, \mathbf{s}_{p q}\right)$ of intensity values which are both dependent on the (discrete) scan position $\mathbf{r}_{j k}=$ $j \delta r \hat{\mathbf{x}}+k \delta r \hat{\mathbf{y}}$ and the (discrete) CCD pixel position $\mathbf{s}_{p q}=$ $p \delta s \hat{\mathbf{x}}+q \delta s \hat{\mathbf{y}}$, where the $j, k, p$, and $q$ are integer numbers, $\delta r$ is the step size of scanning and $\delta s$ the pixel size of the CCD divided by imaging magnification, and the $\hat{\mathbf{x}}$ and $\hat{\mathbf{y}}$ are unit vectors along the horizontal and vertical sample or image direction, respectively. The integration of Eq. (5) has then to be approximated by the sum

$$
I_{\mathrm{eff}}\left(\mathbf{r}_{j k}\right)=\sum_{p, q} I\left(\mathbf{r}_{j k}-\frac{\mathbf{s}_{p q}}{2}, \mathbf{s}_{p q}\right)
$$

where the summation runs over all pixels $(p, q)$ of the region of interest (ROI) that is used on the CCD for recording the images at all scan positions. To perform the summation in Eq. (10), one needs the values of $I$ at double positions $\left(\mathbf{r}_{j k}-\mathbf{s}_{p q} / 2, \mathbf{s}_{p q}\right)$ which are, in general, not contained in the experimentally recorded data set taken at discrete double positions $\left(\mathbf{r}_{j k}, \mathbf{s}_{p q}\right)$. These values have to be calculated from the original data set by using, for example, a cubic interpolation. The resulting effective image $I_{\text {eff }}$ is expected to show already a $\sqrt{2}$ times better resolution than a conventional microscope image. To improve resolution further, the Fourier transform of the effective image $I_{\text {eff }}\left(\mathbf{r}_{j k}\right)$ is weighted with the weight function $\tilde{W}(\mathbf{k})$ as defined by Eq. (9), and the result is backtransformed into real space. The forward and backward Fourier transforms are calculated by using a discrete fast Fourier transform algorithm [10]. As shown in Eq. (9), the weight function $\tilde{W}(\mathbf{k})$ is determined by the PSF $U(\mathbf{r})$ and the EID $E(\mathbf{r})$ (or more precisely, by their Fourier transforms). Both functions can be calculated by using the known optical parameters of the SIM system and assuming ideal optical performance of the system. We calculated both functions using the wave-optical theory developed by Wolf [11] and Richards and Wolf [12] for imaging through optical systems with high numerical aperture. For the EID calculation, we approximated the incident laser beam at the backfocal aperture of the objective by a Gaussian beam with $7 \mathrm{~mm}$ radius, corresponding to the experimental situation and representing a significant overfilling of the back aperture (radius $3.6 \mathrm{~mm}$ ) which results in a diffraction-limited focus size in sample space. The values of the other parameters used were: numerical aperture $\mathrm{NA}=1.2$; refractive index of immersion medium equal to the value of pure water (1.33); excitation and center emission wavelengths 640 and $670 \mathrm{~nm}$, respectively.

We characterized the ISM performance by imaging single fluorescent beads with a size below the diffraction limit of conventional imaging. For this purpose, fluorescent point-spread function beads (TetraSpeck ${ }^{\mathrm{TM}} 100 \mathrm{~nm}$, T7279, Invitrogen, Karlsruhe, Germany) of $100 \mathrm{~nm}$ diameter were immobilized in air on a Lab-Tek chamber slide (177402, Thermo Fischer Scientific/Nunc GmbH \& Co. KG, Langenselbold, Germany) and imaged with the described ISM setup. Sample scanning was performed with the piezo scan mirror of the setup, with $10 \mathrm{~ms}$ acquisition (imaging) time per scan position, total excitation power of the laser of $5 \mu \mathrm{W}$, and scan step size of $40 \mathrm{~nm}$. The image of the excitation region on the EM CCD had a size of less than 16 by 16 pixel, so we restricted intensity recording and readout to a ROI covering this area. Figure 2 shows the image of two close lying beads, where three panels are shown. The left panel corresponds to a conventional CLSM image which was obtained by summing the intensity over the whole recorded ROI of the EM CCD, thus using the EM CCD effectively as a point detector. The middle panel shows the effective image $I_{\text {eff }}$ calculated according to Eq. (10). The right panel shows the Fourier-weighted image. In the image, one can discern not only the central peak of the bead image, but also the surrounding Airy disc. For estimating the resolution improvement, we fitted the two-dimensional intensity distributions with circular Gaussian distributions. A typical linear cross section along the horizontal axis with a Gaussian fit is shown in Fig. 3. We repeated the fit for ten different beads and found amazing homogeneity in peak width. The mean values

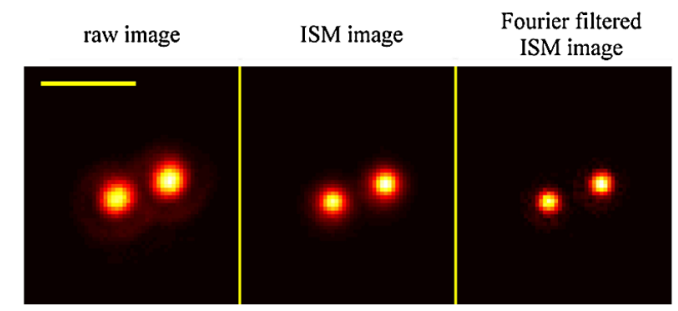

FIG. 2 (color online). Image of a single fluorescent bead of $100 \mathrm{~nm}$ diameter. Left panel: CLSM image; middle panel: ISM image; right panel: Fourier-weighted ISM image. The horizontal bar in the left panel has a length of $1 \mu \mathrm{m}$. 


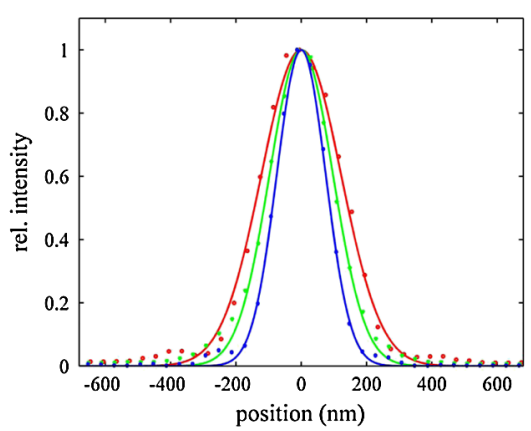

FIG. 3 (color online). Cross section through one of the bead images in Fig. 2.

and standard deviations for the $2 \sigma$ widths of the CLSM, the ISM, and the Fourier-filtered ISM images are $244 \pm 9 \mathrm{~nm}$, $198 \pm 9 \mathrm{~nm}$, and $150 \pm 10 \mathrm{~nm}$, respectively. The total resolution enhancement was $1.63 \pm 0.08$ when comparing the Fourier-filtered ISM image with the CLSM image.

A complete wave-optical modeling of imaging in CLSM, ISM, and Fourier-filtered ISM which takes into account the finite CCD pixel size, the redshift of emission wavelength with respect to excitation wavelength, and the cutoff of Fourier modes as described after Eq. (9), predicts a maximum resolution enhancement factor of less than 1.75. Thus, the measured resolution enhancement is very close to the theoretically maximum possible value, and its slightly lower value is probably due to residual optical aberration of the used optics. Significant advantages of ISM when compared with other methods (such as SIM using wide-field imaging in conjunction with a periodical spatial excitation intensity modulation) are that any existing CLSM can be easily converted into an ISM system, that it is easy to combine ISM with nonlinear optical saturation effects for further improving resolution [13], that extension to multicolor excitation or detection is straightforward, and that ISM keeps the intrinsic 3D-sectioning capability of CLSM. Moreover, combination of ISM with two-photon excitation is also achievable in a straightforward way, thus offering prospects for super-resolution imaging in deep tissue sections.

Financial support by the Human Frontier Science Program (RGP46/2006) and by the German Federal Ministry of Education and Research (FKZ 13N9236) is gratefully acknowledged.

*joerg.enderlein@physik3.gwdg.de

[1] S.W. Hell and A. Schönle, in Biomedical Optical Imaging, edited by J. Fujimoto and D. Farkas (Oxford University Press, Oxford, 2009), pp. 237-264.

[2] S. Hell and A. Schönle, in Science of Microscopy, edited by P. Hawkes and J. Spence (Springer, New York, 2007), Vol. II, pp. 1153-1158.

[3] R. Heintzmann and G. Ficz, Briefings in Functional Genomics and Proteomics 5, 289 (2006).

[4] S. W. Hell, Science 316, 1153 (2007).

[5] B. Huang, M. Bates, and X.W. Zhuang, Annu. Rev. Biochem. 78, 993 (2009).

[6] P. Dedecker, J. Hofkens, and J. I. Hotta, Mater. Today 11, 12 (2008).

[7] M. G. L. Gustafsson, J. Microsc. 198, 82 (2000).

[8] P. Kner, B. B. Chhun, E. R. Griffis, L. Winoto, and M. G. L. Gustafsson, Nat. Methods 6, 339 (2009).

[9] J. Enderlein, E. Toprak, and P. R. Selvin, Opt. Express 14, 8111 (2006).

[10] J. W. Cooley and J. W. Tukey, Math. Comput. 19, 297 (1965).

[11] E. Wolf, Proc. R. Soc. A 253, 349 (1959).

[12] B. Richards and E. Wolf, Proc. R. Soc. A 253, 358 (1959).

[13] M. G. L. Gustafsson, Proc. Natl. Acad. Sci. U.S.A. 102, 13081 (2005). 